\title{
Pacific
}

Journal of

Mathematics

\section{DETERMINANT IDENTITIES}

George W. Eyre Andrews And William H. Burge 


\section{DETERMINANT IDENTITIES}

\section{George E. Andrews and William H. Burge}

A number of determinants are evaluated in closed form including

$$
\operatorname{det}\left(\left(\begin{array}{c}
i+j+x \\
2 i-j
\end{array}\right)+\left(\begin{array}{c}
i+j+y \\
2 i-j
\end{array}\right)\right)_{0 \leq i, j \leq n-1} .
$$

1. Introduction. In one of their series of papers on plane partitions and related questions, Mills, Robbins and Rumsey [9; p. 53] prove the following determinant formula.

$$
m_{n}(x)=\operatorname{det}\left(\left(\begin{array}{c}
i+j+x \\
2 i-j
\end{array}\right)\right)_{0 \leq i, j \leq n-1}=\frac{1}{2^{n}} \prod_{k=0}^{n-1} \Delta_{2 k}(2 x),
$$

where $\Delta_{0}(u)=2$ and for $j>0$

$$
\Delta_{2 j}(u)=\frac{(u+2 j+2)_{j}\left(\frac{1}{2} u+2 j+\frac{3}{2}\right)_{j-1}}{(j)_{j}\left(\frac{1}{2} u+j+\frac{3}{2}\right)_{j-1}}
$$

with

$$
(A)_{j}=A \cdot(A+1) \cdots(A+j-1) .
$$

Our object here is primarily to prove the following generalization of (1.1).

THEOREM 1. Let

$$
\begin{gathered}
M_{n}(x, y)=\operatorname{det}\left(\left(\begin{array}{c}
i+j+x \\
2 i-j
\end{array}\right)+\left(\begin{array}{c}
i+j+y \\
2 i-j
\end{array}\right)\right)_{0 \leq i, j \leq n-1} \\
N_{n}(x, y)=\operatorname{det}\left(\frac { 2 } { x + 1 - y } \left\{\left(\begin{array}{c}
i+j+x+1 \\
2 i-j+1
\end{array}\right)\right.\right. \\
\left.\left.\quad-\left(\begin{array}{c}
i+j+y \\
2 i-j+1
\end{array}\right)\right\}\right)_{0 \leq i, j \leq n-1}
\end{gathered}
$$

Then

$$
M_{n}(x, y)=N_{n}(x, y)=\prod_{k=0}^{n-1} \Delta_{2 k}(x+y)
$$


Sections 2 and 3 will be devoted to the proof of this result. In $\S \S 4$ and 5 we shall show how our work leads to two alternative proofs of the T.S.S.C.P.P. conjecture [2], and we shall mention a related application of Ishikawa [6].

2. Bailey's balanced ${ }_{4} F_{3}$ summation. In this section, we consider summation formulas for hypergeometric series [4; p. 8], [10, p. 41]:

$$
{ }_{n+1} F_{n}\left[\begin{array}{c}
a_{0}, a_{1}, \ldots, a_{n} ; t \\
b_{1}, \ldots, b_{n}
\end{array}\right]=\sum_{j=0}^{\infty} \frac{\left(a_{0}\right)_{j}\left(a_{1}\right)_{j} \cdots\left(a_{n}\right)_{j} t^{n}}{j !\left(b_{1}\right)_{j} \cdots\left(b_{n}\right)_{j}}
$$

The formula of Bailey [3; p. 512, (c)] [10; p. 245, (III.20)] alluded to in the title of this section is

$$
{ }_{4} F_{3}\left[\begin{array}{c}
\frac{a}{2}, \frac{a+1}{2}, b+n,-n ; 1 \\
\frac{b}{2}, \frac{b+1}{2}, a+1
\end{array}\right]=\frac{(b-a)_{n}}{(b)_{n}},
$$

and a closely related companion is

$$
{ }_{4} F_{3}\left[\begin{array}{c}
\frac{a}{2}, \frac{a+1}{2}, b+n,-n ; 1 \\
\frac{b+1}{2}, \frac{b+2}{2}, a
\end{array}\right]=\frac{(b-a+1)_{n}}{(b+1)_{n-1}(b+2 n)} .
$$

Also useful for our work is a transformation due to F.J.W. Whipple [14; p. 537, eq. (10.1)]. If one of $z$ and $n$ is a nonnegative integer, then

$$
\begin{aligned}
{ }_{4} F_{3}\left[\begin{array}{c}
a, b,-z,-n ; 1 \\
u, v, w
\end{array}\right] \\
=\frac{\Gamma(v+z+n) \Gamma(w+z+n) \Gamma(v) \Gamma(w)}{\Gamma(v+z) \Gamma(v+n) \Gamma(w+n) \Gamma(w+z)} \\
\quad \times{ }_{4} F_{3}\left[\begin{array}{c}
u-a, u-b,-z,-n ; 1 \\
1-v-z-n, 1-w-z-n, u
\end{array}\right] .
\end{aligned}
$$

We note in passing that (2.4) specialized to $a=\alpha / 2, b=(\alpha+1) / 2$, $z=-\beta-\nu, n=\nu, v=(\beta+1) / 2, w=(\beta+2) / 2, u=\alpha$ yields

$$
\begin{aligned}
& { }_{4} F_{3}\left[\begin{array}{c}
\frac{\alpha}{2}, \frac{\alpha+1}{2}, \beta+\nu,-\nu ; 1 \\
\frac{\beta+1}{2}, \frac{\beta+2}{2}, \alpha
\end{array}\right] \\
& =\frac{\beta}{\beta+2 \nu}{ }_{4} F_{3}\left[\begin{array}{c}
\frac{\alpha-1}{2}, \frac{\alpha}{2}, \beta+\nu,-\nu ; 1 \\
\frac{\beta+1}{2}, \frac{\beta}{2}, 1+(\alpha-1)
\end{array}\right] \\
& =\frac{\beta}{\beta+2 \nu} \frac{(\beta-\alpha+1)_{\nu}}{(\beta)_{\nu}} \quad(\text { by }(2.2)) \text {. }
\end{aligned}
$$

Thus (2.3) is an immediate consequence of (2.2) and (2.4).

LEMMA 1. Let $\alpha$ be a positive integer, then

$$
{ }_{4} F_{3}\left[\begin{array}{c}
-\frac{\alpha}{2},-\frac{\alpha-1}{2}, b+z,-z ; 1 \\
\frac{b}{2}, \frac{b+1}{2}, 1-\alpha
\end{array}\right]=\frac{(b+z)_{\alpha}}{(b)_{\alpha}}+\frac{(-z)_{\alpha}}{(b)_{\alpha}} .
$$


Proof. We begin by considering (2.2) when $a=-\alpha$ a negative integer. The index of summation $j$ runs from 0 to $n$ (when $j>$ $n$ all terms $=0)$. Furthermore, the terms with $\alpha / 2<j<\alpha$ are all identically zero. For $n \geq j \geq \alpha$ there are cancelling zeros in numerator and denominator.

Thus by (2.2)

$$
\begin{aligned}
\frac{(b+\alpha)_{n}}{(b)_{n}}= & \sum_{0 \leq 2 j \leq \alpha} \frac{\left(-\frac{\alpha}{2}\right)_{J}\left(\frac{1-\alpha}{2}\right)_{J}(b+n)_{J}(-n)_{J}}{j !\left(\frac{b}{2}\right)_{j}\left(\frac{b+1}{2}\right)_{j}(1-\alpha)_{j}} \\
& +\sum_{j=\alpha}^{n} \frac{(-\alpha)(-\alpha+1) \cdots(-1)(1)(2) \cdots(-\alpha+2 j-1)(b+n)_{j}(-n)_{J}}{j !(b)_{2 j}(1-\alpha)(2-\alpha) \cdots(-1) \cdot(1) \cdot(2) \cdots(j-\alpha)} \\
= & S_{1}+S_{2} .
\end{aligned}
$$

Now

$$
\begin{aligned}
S_{2} & =\sum_{j=0}^{n-\alpha} \frac{(-1)^{\alpha} \alpha !(\alpha+2 j-1) !(b+n)_{j+\alpha}(-n)_{j+\alpha}}{(j+\alpha) !(b)_{2 j+2 \alpha}(-1)^{\alpha-1}(\alpha-1) ! j !} \\
& =-\frac{(b+n)_{\alpha}(-n)_{\alpha}}{(b)_{2 \alpha}} \sum_{j=0}^{n-\alpha} \frac{(\alpha)_{2 j}(b+n+\alpha)_{j}(-n+\alpha)_{j}}{j !(b+2 \alpha)_{2 j}(\alpha+1)_{j}} \\
& =-\frac{(b+n)_{\alpha}(-n)_{\alpha}}{(b)_{2 \alpha}}{ }_{4} F_{3}\left(\begin{array}{c}
\left.\frac{\alpha}{2}, \frac{\alpha+1}{2}, b+2 \alpha+(n-\alpha),-(n-\alpha) ; 1\right) \\
\frac{b+2 \alpha}{2}, \frac{b+2 \alpha+1}{2}, 1+\alpha
\end{array}\right) \\
& =-\frac{(b+n)_{\alpha}(-n)_{\alpha}}{(b)_{2 \alpha}} \frac{(b+\alpha)_{n-\alpha}}{(b+2 \alpha)_{n-\alpha}} \quad(\text { by }(2.2)) \\
& =-\frac{(-n)_{\alpha}}{(b)_{\alpha}} .
\end{aligned}
$$

\section{Hence}

$$
\begin{aligned}
\sum_{0 \leq 2 j \leq \alpha} & \frac{\left(-\frac{\alpha}{2}\right)_{j}\left(\frac{1-\alpha}{2}\right)_{j}(b+n)_{j}(-n)_{j}}{j !\left(\frac{b}{2}\right)_{j}\left(\frac{b+1}{2}\right)_{j}(1-\alpha)_{j}} \\
= & \frac{(b+\alpha)_{n}}{(b)_{n}}+\frac{(-n)_{\alpha}}{(b)_{\alpha}}=\frac{(b+n)_{\alpha}}{(b)_{\alpha}}+\frac{(-n)_{\alpha}}{(b)_{\alpha}},
\end{aligned}
$$

which is precisely (2.6) when $z$ is any positive integer, $n$. However, both sides of (2.6) are polynomials in $z$ of degree at most $\alpha$, and since they agree for all positive integral $z$ we see that (2.6) holds for all real $z$. 
LEMMA 2. Let $\alpha$ be a nonnegative integer, then

$$
\begin{aligned}
{ }_{4} F_{3}\left[\begin{array}{c}
-\frac{\alpha}{2}, \frac{-\alpha+1}{2}, b+z,-z ; 1 \\
\frac{b+1}{2}, \frac{b+2}{2},-\alpha
\end{array}\right] \\
=\frac{(b+z)_{\alpha+1}}{(b+2 z)(b+1)_{\alpha}}-\frac{(-z)_{\alpha+1}}{(b+1)_{\alpha}(b+2 z)} .
\end{aligned}
$$

Proof. In parallel with Lemma 1, we begin by considering (2.3) with $a=-\alpha$ a negative integer. If $j$ is the index of summation in the ${ }_{4} F_{3}$, then the nonzero terms of the sum occur for $0 \leq j \leq \alpha / 2$ and $\alpha<j \leq n$. If we call the two resulting sums $T_{1}$ and $T_{2}$, then

$$
\frac{(b+\alpha+1)_{n}}{(b+1)_{n-1}(b+2 n)}=T_{1}+T_{2} \text {. }
$$

Now

$$
\begin{aligned}
T_{2}= & \sum_{j=\alpha+1}^{n} \frac{(-\alpha)_{\alpha}(1)_{2 j-\alpha-1}(b+n)_{j}(-n)_{j}}{j !(b+1)_{2 j}(-\alpha)_{\alpha}(1)_{j-\alpha-1}} \\
= & \sum_{j=0}^{n-\alpha-1} \frac{(1)_{2 j+\alpha+1}(b+n)_{j+\alpha+1}(-n)_{j+\alpha+1}}{(j+\alpha+1) !(b+1)_{2 j+2 \alpha+2} j !} \\
= & \frac{(\alpha+1) !(b+n)_{\alpha+1}(-n)_{\alpha+1}}{(\alpha+1) !(b+1)_{2 \alpha+2}} \\
& \times{ }_{4} F_{3}\left[\frac{\alpha}{2}+1, \frac{\alpha}{2}+\frac{3}{2}, b+n+\alpha+1,-n+\alpha+1 ; 1\right] \\
= & \frac{(b+1)_{\alpha+n}(-n)_{\alpha+1}}{(b+1)_{n-1}(b+1)_{2 \alpha+2}} \frac{(b+\alpha+1)_{n-\alpha-1}}{(b+2 \alpha+3)_{n-\alpha-2}(b+2 n)} \\
= & \frac{(-n)_{\alpha+1}}{(b+1)_{\alpha}(b+2 n)} .
\end{aligned}
$$

Hence

$$
\begin{aligned}
& { }_{4} F_{3}\left[\begin{array}{c}
-\frac{\alpha}{2}, \frac{-\alpha+1}{2}, b+n,-n ; 1 \\
\frac{b+1}{2}, \frac{b+2}{2},-\alpha
\end{array}\right] \\
& =T_{1}=\frac{(b+\alpha+1)_{n}}{(b+1)_{n-1}(b+2 n)}-\frac{(-n)_{\alpha+1}}{(b+1)_{\alpha}(b+2 n)} \\
& =\frac{(b+n)_{\alpha+1}}{(b+1)_{\alpha}(b+2 n)}-\frac{(-n)_{\alpha+1}}{(b+1)_{\alpha}(b+2 n)} \text {, }
\end{aligned}
$$

which is (2.8) when $z$ is any positive integer. Since (2.8) is an identity of rational functions in $z$, the result in full generality follows immediately. 
3. The main theorem. Our proof of Theorem 1 relies on the following binomial coefficient summations.

Lemma 3. For integers $i, j \geq 0$

$$
\begin{gathered}
2 \sum_{k=0}^{i} \frac{(y-x)}{(y-x+i-k)}\left(\begin{array}{c}
y-x+i-k \\
2 i-2 k
\end{array}\right)\left(\begin{array}{c}
k+j+x+y \\
2 k-j
\end{array}\right) \\
\quad=\left(\begin{array}{c}
i+j+2 x \\
2 i-j
\end{array}\right)+\left(\begin{array}{c}
i+j+2 y \\
2 i-j
\end{array}\right) .
\end{gathered}
$$

$$
\begin{gathered}
\sum_{k=0}^{i} \frac{(2 x-2 y+1)}{(y-x+i-k)}\left(\begin{array}{c}
y-x+i-k \\
2 i-2 k+1
\end{array}\right)\left(\begin{array}{c}
k+j+x+y \\
2 k-j
\end{array}\right) \\
=\left(\begin{array}{c}
i+j+2 x+1 \\
2 i-j+1
\end{array}\right)-\left(\begin{array}{c}
i+j+2 y \\
2 i-j+1
\end{array}\right) .
\end{gathered}
$$

Proof. We note that the only nonzero terms on the left-hand side of (3.1) occur for $i \geq k \geq j / 2$. Consequently, if $j>2 i$ then both sides of (3.1) are zero. Hence we may assume $2 i \geq j$.

\section{Therefore}

$$
\begin{aligned}
2 \sum_{k=0}^{i} & \frac{(y-x)}{(y-x+i-k)}\left(\begin{array}{c}
y-x+i-k \\
2 i-2 k
\end{array}\right)\left(\begin{array}{c}
k+j+x+y \\
2 k-j
\end{array}\right) \\
= & 2 \sum_{k=0}^{i} \frac{(y-x)}{(y-x+k)}\left(\begin{array}{c}
y-x+k \\
2 k
\end{array}\right)\left(\begin{array}{c}
i+j-k+x+y \\
2 i-j-2 k
\end{array}\right) \\
= & 2\left(\begin{array}{c}
i+j+x+y \\
2 i-j
\end{array}\right) \sum_{k=0}^{j} \frac{(y-x)_{k}(-y+x)_{k}(-2 i+j)_{2 k}}{(2 k) !(-i-j-x-y)_{k}(2 j-i+x+y+1)_{k}} \\
= & 2\left(\begin{array}{c}
i+j+x+y \\
2 i-j
\end{array}\right){ }_{4} F_{3}\left[\begin{array}{c}
y-x,-y+x,-i+\frac{j}{2},-i+\frac{j+1}{2} ; 1 \\
\frac{1}{2},-i-j-x-y, 2 j-i+x+y+1
\end{array}\right] \\
= & 2\left(\begin{array}{c}
i+j+x+y \\
2 i-j
\end{array}\right) \quad \frac{\Gamma(i+j+x+y+1 / 2) \Gamma(2 i-j) \Gamma(2 j-i+x+y+1) \Gamma(1 / 2)}{\Gamma(3 j / 2+x+y+1) \Gamma(1 / 2+i-j / 2) \Gamma(3 j / 2+x+y+1 / 2) \Gamma(i-j / 2)} \\
& \times{ }_{4} F_{3}\left[\begin{array}{c}
-i-j-2 y,-i-j-2 x,-i+\frac{j}{2},-i+\frac{j+1}{2} ; 1 \\
-j-i-x-y+\frac{1}{2},-i-j-x-y, 1-2 i+j
\end{array}\right] \quad(\mathrm{by}(2.4)) \\
= & \left(\begin{array}{c}
i+j+x+y \\
2 i-j
\end{array}\right) \frac{\Gamma(i+j+x+y+1 / 2) \Gamma(2 j-i+x+y+1) 2^{2 i+2 j+2 x+2 y}}{\Gamma(1 / 2) \Gamma(3 j+2 x+2 y+1)} \\
& \times\left(\begin{array}{c}
(-i-j-2 x)_{2 l-j} \\
(-2 i-2 j-2 x-2 y)_{2 i-j}+\frac{(-i-j-2 y)_{2 i-j}}{(-2 i-2 j-2 x-2 y)_{2 i-j}}
\end{array}\right)
\end{aligned}
$$

(by Lemma 1 and Gauss's duplication formula [5; p. 5, eq. (15)]) $=\left(\begin{array}{c}i+j+2 x \\ 2 i-j\end{array}\right)+\left(\begin{array}{c}i+j+2 y \\ 2 i-j\end{array}\right)$

as desired for (3.1). 
For (3.2) again we obtain zero equals zero unless $2 i \geq j$. Hence

$$
\begin{aligned}
& \sum_{k=0}^{i} \frac{(2 x-2 y+1)}{(y-x+i-k)}\left(\begin{array}{c}
y-x+i-k \\
2 i-2 k+1
\end{array}\right)\left(\begin{array}{c}
k+j+x+y \\
2 k-j
\end{array}\right) \\
& =\sum_{k=0}^{i} \frac{(2 x-2 y+1)}{(y-x+k)}\left(\begin{array}{c}
y-x+k \\
2 k+1
\end{array}\right)\left(\begin{array}{c}
i-k+j+x+y \\
2 i-j-2 k
\end{array}\right) \\
& =(2 x-2 y+1)\left(\begin{array}{c}
i+j+x+y \\
2 i-j
\end{array}\right) \\
& \times{ }_{4} F_{3}\left[\begin{array}{c}
y-x, 1+x-y,-i+\frac{j}{2},-i+\frac{j}{2}+\frac{1}{2} ; 1 \\
\frac{3}{2},-i-j-x-y, x+y+2 j-i+1
\end{array}\right] \\
& =(2 x-2 y+1)\left(\begin{array}{c}
i+j+x+y \\
2 i-j
\end{array}\right) \\
& \times \frac{\Gamma(2 i-j+1) \Gamma(i+j+x+y+1 / 2) \Gamma(3 / 2) \Gamma(2 j-i+x+y+1)}{\Gamma\left(i-\frac{j}{2}+\frac{3}{2}\right) \Gamma\left(i-\frac{j}{2}+1\right) \Gamma\left(\frac{3 j}{2}+x+y+1\right) \Gamma\left(\frac{3 j}{2}+x+y+\frac{1}{2}\right)} \\
& \times{ }_{4} F_{3}\left[\begin{array}{c}
-i-j-2 y,-i-j-2 x-1,-i+\frac{1}{2},-i+\frac{j}{2}+\frac{1}{2} ; 1 \\
-i-j-x-y,-2 i+j,-i-j-x-y+\frac{1}{2}
\end{array}\right] \\
& =(2 x-2 y+1)\left(\begin{array}{c}
i+j+x+y \\
2 i-j
\end{array}\right) \\
& \times \frac{\Gamma\left(i+j+x+y+\frac{1}{2}\right) 2^{2 l+2 j+2 x+2 y} \Gamma(2 j-i+x+y+1)}{\Gamma\left(\frac{1}{2}\right) \Gamma(3 j+2 x+2 y+1)(2 i-j+1)} \\
& \times\left(\frac{(-i-j-2 x-1)_{2 l-j+1}}{(2 y-2 x-1)(-2 i-2 j-2 x-2 y)_{2 i-j}}\right. \\
& \left.-\frac{(-i-j-2 y)_{2 l-j+1}}{(2 y-2 x-1)(-2 i-2 j-2 x-2 y)_{2 i-j}}\right) \\
& =\left(\begin{array}{c}
i+j+2 x+1 \\
2 i-j+1
\end{array}\right)-\left(\begin{array}{c}
i+j+2 y \\
2 i-j+1
\end{array}\right) \text {. }
\end{aligned}
$$

Proof of Theorem 1. We define five matrices

$$
\mathbf{M}_{n}(x)=\left(\left(\begin{array}{c}
i+j+x \\
2 i-j
\end{array}\right)\right)_{0 \leq i, j \leq n-1}
$$

$$
\mu_{n}(x, y)=\left(\left(\begin{array}{c}
i+j+x \\
2 i-j
\end{array}\right)+\left(\begin{array}{c}
i+j+y \\
2 i-j
\end{array}\right)\right)_{0 \leq i, j \leq n-1}
$$

(3.7) $\nu_{n}(x, y)=\left(\frac{2}{x+1-y}\left(\left(\begin{array}{c}i+j+x+1 \\ 2 i-j+1\end{array}\right)\right.\right.$

$$
\left.\left.-\left(\begin{array}{c}
i+j+y \\
2 i-j+1
\end{array}\right)\right)\right)_{0 \leq i, j \leq n-1}
$$

$$
\tau_{n}(x, y)=\left(\frac{2(y-x)}{(y-x+i-j)}\left(\begin{array}{c}
y-x+i-j \\
2 i-2 j
\end{array}\right)\right)_{0 \leq i, j \leq n-1}
$$


(3.9)

$$
\sigma_{n}(x, y)=\left(\frac{2}{(y-x+i-j)}\left(\begin{array}{c}
y-x+i-j \\
2 i-2 j+1
\end{array}\right)\right)_{0 \leq i, j \leq n-1}
$$

Clearly

$$
m_{n}(x)=\operatorname{det}\left(\mathbf{M}_{n}(x)\right)
$$

$$
M_{n}(x, y)=\operatorname{det}\left(\mu_{n}(x, y)\right),
$$

$$
N_{n}(x, y)=\operatorname{det}\left(\nu_{n}(x, y)\right) \text {, }
$$

and

$$
\operatorname{det}\left(\tau_{n}(x, y)\right)=2^{n},
$$

Equations (3.10)-(3.12) are restatements of (1.1), (1.4) and (1.5), while (3.13) and (3.14) are obvious since each matrix in question is lower triangular.

Now

$$
\operatorname{det}\left(\sigma_{n}(x, y)\right)=2^{n} .
$$


Similarly

(3.16) $\sigma_{n}(x, y) \mathbf{M}_{n}(x+y)$

$$
\begin{aligned}
& =\left(\sum_{k=0}^{i} \frac{2}{(y-x+i-k)}\left(\begin{array}{c}
y-x+i-k \\
2 i-2 k+1
\end{array}\right)\left(\begin{array}{c}
k+j+x+y \\
2 k-j
\end{array}\right)\right)_{0 \leq i, j \leq n-1} \\
& =\left(\frac{2}{(2 x-2 y+1)}\left(\left(\begin{array}{c}
i+j+2 x+1 \\
2 i-j+1
\end{array}\right)-\left(\begin{array}{c}
i+j+2 y \\
2 i-j+1
\end{array}\right)\right)\right)_{0 \leq i, j \leq n-1} \\
& \quad \text { (by Lemma 3, eq. (3.2)) }
\end{aligned}
$$

Therefore by (3.16) and (1.1)

$$
\begin{aligned}
N_{n}(x, y) & =\operatorname{det}\left(\nu_{n}(x, y)\right) \\
& =\operatorname{det}\left(\sigma_{n}\left(\frac{x}{2}, \frac{y}{2}\right) \mathbf{M}_{n}\left(\frac{x+y}{2}\right)\right) \\
& =2^{n} \cdot \operatorname{det}\left(\mathbf{M}_{n}\left(\frac{x+y}{2}\right)\right) \\
& =\prod_{k=0}^{n-1} \Delta_{2 k}(x+y) .
\end{aligned}
$$

4. Applications to the T.S.S.C.P.P. conjecture. We shall consider in this section a few instances of the results we have obtained. We begin with a rather odd determinant for generalized harmonic numbers.

COROllaRY 1. Let $H_{n}(x)=\sum_{j=0}^{n} \frac{1}{x+j}$, then

$$
\begin{aligned}
& \operatorname{det}\left(\left(\begin{array}{c}
i+j+x \\
2 i-j+1
\end{array}\right)\left(H_{i+j}(x)-H_{2 j-i-1}(x)\right)\right)_{0 \leq i, j \leq n-1} \\
& \quad=\frac{1}{2^{n}} \prod_{k=0}^{n-1} \Delta_{2 k}(2 x-1) .
\end{aligned}
$$

Proof. From (1.5) and (1.6) with $x$ replaced by $x-1$ we find

$$
\begin{aligned}
& \operatorname{det}\left(\frac{1}{x-y}\left(\left(\begin{array}{c}
i+j+x \\
2 i-j+1
\end{array}\right)-\left(\begin{array}{c}
i+j+y \\
2 i-j+1
\end{array}\right)\right)\right)_{0 \leq i, j \leq n-1} \\
& \quad=2^{-n} \prod_{k=0}^{n-1} \Delta_{2 k}(x+y-1) .
\end{aligned}
$$

Now let $y \rightarrow x$, and we obtain

$$
\operatorname{det}\left(\frac{d}{d x}\left(\begin{array}{c}
i+j+x \\
2 i-j+1
\end{array}\right)\right)_{0 \leq i, j \leq n-1}=2^{-n} \prod_{k=0}^{n-1} \Delta_{2 k}(2 x-1)
$$


Identity (4.1) is merely (4.3) after the differentiation has been completed.

In [8], Mills, Robbins and Rumsey define "a totally symmetric plane partition of size $n$ (to be) a plane partition whose three-dimensional Ferrers graph is contained in the box

$$
X_{n}=[1, n] \times[1, n] \times[1, n]
$$

and which is mapped to itself under all permtutations of the coordinate axes. The complement of the Ferrers graph of such a plane partition (that is, the set of lattice points in the box $X_{n}$ that do not belong to the Ferrers graph) is again totally symmetric when viewed from the vantage point of the vertex $(n+1, n+1, n+1)$. A totally symmetric plane partition is self complementary if it is congruent (in the geometrical sense) to its complement. This cannot occur unless $n=2 m$ is even".

If we define $A_{n}$ by the recurrence (4.6), then Mills et al. [8] conjecture that $A_{n}$ is the total number of TSSCPP's in $X_{2 n}$.

In [12], J. Stembridge essentially proved that the TSSCPP conjecture reduces to the following result (the details of Stembridge's result and its equivalence to the following are provided in [2; Sec. 2]). It should be noted that our proof of Corollary 2 in the final analysis relies on (1.1) and thus is quite different from the proof of the T.S.S.C.P.P. Conjecture given in [2].

Corollary 2.

$$
\operatorname{det}\left(a_{i j}\right)_{0 \leq i, j \leq n-1}=A_{n}^{2},
$$

where

$$
a_{i j}= \begin{cases}1 & \text { if } i=j=0, \\
0 & \text { if } i=j>0, \\
\sum_{s=2 i-j+1}^{2 j-i}\left(\begin{array}{c}
i+j \\
-a_{j i}
\end{array}\right) & \text { if } i<j, \\
& \text { if } i>j,\end{cases}
$$

and

$$
A_{n}=\prod_{i=0}^{n-1} \frac{(3 i+1) !}{(n+i) !}=\frac{(3 n-2) !(n-1) !}{(2 n-1) !(2 n-2) !} A_{n-1}
$$


Proof. We define several new matrices:

$$
w(n)=\left(\left(\begin{array}{c}
i+j+1 \\
2 j-i
\end{array}\right)+\left(\begin{array}{c}
i+j \\
2 j-i-1
\end{array}\right)\right)_{0 \leq i, j \leq n-1},
$$

$$
u(n)=\left(\delta_{i j}-2 \delta_{i, j+1}\right)_{0 \leq i, j \leq n-1},
$$

(4.9) $v(n)=\left(\left(\begin{array}{c}i+j+2 \\ 2 j-i+1\end{array}\right) \frac{(3 i+1)(3 j+1)(3 j-3 i)}{(i+j)(i+j+1)(i+j+2)}\right)_{0 \leq i, j \leq n-1}$

(where we define the $(0,0)$-th entry of $v(n)$ to be 1 ),

$$
\begin{aligned}
u_{1}(n) & =u(n)+\left(2 \delta_{(i-1)^{2}+j, 0}\right)_{0 \leq i, j \leq n-1} \\
& =\left(\begin{array}{ccccc}
1 & 0 & 0 & 0 & \cdots \\
0 & 1 & 0 & 0 & \cdots \\
0 & -2 & 1 & 0 & \cdots \\
0 & 0 & -2 & 1 & \cdots
\end{array}\right)_{n \times n}
\end{aligned}
$$

$$
s t(n)=\left(a_{i j}\right)_{0 \leq i, j \leq n-1} .
$$

The matrices $u$ and $u_{1}$ are introduced to perform certain simple row and column operations. In particular, elementary algebra reveals that

$$
u(n) w(n)=v(n)
$$

and

$$
u_{1}(n) \operatorname{st}(n)\left(u_{1}(n)\right)^{T}=v(n)
$$

Finally, if we expand $M_{n+1}(-2,-1)$ along the top row we find (4.14) $M_{n+1}(-2,-1)$

$$
\begin{aligned}
& =2 \operatorname{det}\left(\left(\begin{array}{c}
i+j \\
2 i-j+1
\end{array}\right)+\left(\begin{array}{c}
i+j+1 \\
2 i-j+1
\end{array}\right)\right)_{0 \leq i, j \leq n-1} \\
& =2 \operatorname{det}\left(\left(\begin{array}{c}
i+j \\
2 j-i-1
\end{array}\right)+\left(\begin{array}{c}
i+j+1 \\
2 j-i
\end{array}\right)\right)_{0 \leq i, j \leq n-1} \\
& \quad\left(\operatorname{since}\left(\begin{array}{l}
A \\
B
\end{array}\right)=\left(\begin{array}{c}
A \\
A-B
\end{array}\right)\right) \\
& =2 \operatorname{det}(w(n)) .
\end{aligned}
$$


Consequently, since the determinants of $u$ and $u_{1}$ are each 1 , it follows from (1.6), (4.12), (4.13) and (4.14) that

$$
\begin{aligned}
\operatorname{det}\left(a_{i j}\right)_{0 \leq i, j \leq n-1} & =\operatorname{det}(\operatorname{st}(n))=\operatorname{det}(v(n)) \\
& =\operatorname{det}(w(n))=\frac{1}{2} M_{n+1}(-2,-1) \\
& =\prod_{k=1}^{n} \Delta_{2 k}(-3)=A_{n}^{2}
\end{aligned}
$$

because setting $u=-3$ in (1.2) we find

$$
\Delta_{2 k}(-3)=\left(\frac{(3 k-2) !(k-1) !}{(2 k-2) !(2 k-1) !}\right)^{2} .
$$

5. A related identity and another proof of the T.S.S.C.P.P. conjecture. We have not found any related identities for two variables that are genuinely different from (1.6). However, there is one with one variable that merits mention. To this end we need the $\Delta_{j}(n)$ with odd subscript [1; p. 196]:

$$
\Delta_{2 j-1}(\mu)=\frac{(\mu+2 j)_{j-1}\left(\frac{1}{2} \mu+2 j+\frac{1}{2}\right)_{j}}{(j)_{j}\left(\frac{1}{2} \mu+j+\frac{1}{2}\right)_{j-1}} .
$$

The next formula is implicit in the work of Mills-Robbins-Rumsey [9]. However, they do not state it so we record it here: Let

$$
P_{n}(\mu)=\left(\left(\begin{array}{c}
i+j+\mu \\
2 i-j
\end{array}\right)+2\left(\begin{array}{c}
i+j+\mu+2 \\
2 i-j+1
\end{array}\right)\right)_{0 \leq i, j \leq n-1} .
$$

In the notation of [9; p. 50], the determinant of $P_{n}(\mu)$ is $R_{n}(1, \mu)$. This is easily seen by setting $x=1$ in their definition of $R_{n}(x, \mu)$ [9; p. 50] and applying the Chu-Vandermonde summation. Furthermore, from their Theorems 5 and 7, it is easy to see that

$$
\begin{aligned}
\operatorname{det}\left(P_{n}(\mu)\right) & =\frac{\operatorname{det}\left(\delta_{i j}+\left(\begin{array}{c}
i+j+2 \mu \\
i
\end{array}\right)\right)_{0 \leq i, j \leq 2 n-1}}{\operatorname{det}\left(\left(\begin{array}{c}
i+j+\mu \\
2 i-j
\end{array}\right)\right)_{0 \leq i, j \leq n-1}} \\
& =\frac{2^{n} \prod_{k=0}^{2 n-1} \Delta_{k}(2 \mu)}{\prod_{k=0}^{n-1} \Delta_{2 k}(2 \mu)}=2^{n} \prod_{k=1}^{n} \Delta_{2 k-1}(2 \mu) .
\end{aligned}
$$

Our final result gives the determinant for

$$
W_{n}(x)=\left(\left(\begin{array}{c}
i+j+x+1 \\
2 i-j
\end{array}\right)+\left(\begin{array}{c}
i+j+x \\
2 i-j-1
\end{array}\right)\right)_{1 \leq i, j \leq n} .
$$




\section{THEOREM 2.}

$$
\operatorname{det}\left(W_{n}(x)\right)=\prod_{k=1}^{n} \Delta_{2 k-1}(2 x+3) .
$$

Proof. We require an auxiliary matrix

$$
\mathscr{S}_{n}(x)=\left(\frac{(-1 / 2)_{i-j}(-1)^{i-j}}{2^{2 i-2 j-1}(i-j) !}\right)_{0 \leq i, j \leq n-1} .
$$

Consequently,

$$
\mathscr{S}_{n}(x) \cdot W_{n}(x)=P_{n}(x+3 / 2) .
$$

This is easily seen if we introduce

$$
f(i, j, X)=\sum_{k=0}^{i} \frac{(-1 / 2)_{i-k}(-1)^{i-k}}{2^{2 i-2 k-1}(i-k) !}\left(\begin{array}{c}
k+j+X \\
2 k-j
\end{array}\right),
$$

and note that as an immediate corollary of the Pfaff-Saalschultz summation $[4 ;$ p. 9]:

$$
f(i, j, X)=\left(\begin{array}{c}
X+i+j-1 / 2 \\
2 i-j
\end{array}\right)+\left(\begin{array}{c}
X+i+j+1 / 2 \\
2 i-j
\end{array}\right) .
$$

Thus

$$
\begin{aligned}
& \mathscr{S}_{n}(x) W_{n}(x)\left(\sum _ { k \geq 0 } \frac { ( - 1 / 2 ) _ { i - k } ( - 1 ) ^ { i - k } } { 2 ^ { 2 i - 2 k - 1 } ( i - k ! } \left\{\left(\begin{array}{c}
k+j+x+3 \\
2 k-j+1
\end{array}\right)\right.\right. \\
&\left.\left.\quad+\left(\begin{array}{c}
k+j+x+2 \\
2 k-j
\end{array}\right)\right\}\right)_{0 \leq i, j \leq n-1} \\
&=(f(i, j-1, x+4)+f(i, j, x+2))_{0 \leq i, j \leq n-1} \\
&=\left(\left(\begin{array}{c}
x+i+j+5 / 2 \\
2 i-j+1
\end{array}\right)+\left(\begin{array}{c}
x+i+j+7 / 2 \\
2 i-j+1
\end{array}\right)\right. \\
&\left.\quad+\left(\begin{array}{c}
x+i+j+3 / 2 \\
2 i-j
\end{array}\right)+\left(\begin{array}{c}
x+i+j+5 / 2 \\
2 i-j
\end{array}\right)\right)_{0 \leq i, j \leq n-1} \\
&\left.=\left(\begin{array}{c}
x+i+j+7 / 2 \\
2 i-j+1
\end{array}\right)+\left(\begin{array}{c}
x+i+j+3 / 2 \\
2 i-j
\end{array}\right)\right)_{0 \leq i, j \leq n-1} \\
&=P_{n}(x+3 / 2),
\end{aligned}
$$


as asserted in (5.7). Consequently since $\operatorname{det}\left(\mathscr{S}_{n}(x)\right)=2^{n}$, we see from (5.7) and (5.3) that

$$
\operatorname{det}\left(W_{n}(x)\right)=\prod_{k=1}^{n} \Delta_{2 k-1}(2 x+3)
$$

as asserted in Theorem 2 .

We note that Corollary 2 is also derivable from Theorem 2. This is because from (4.7), (5.4) and (5.5)

$$
\operatorname{det}(w(n))=\operatorname{det}\left(W_{n-1}(0)\right)=\prod_{k=1}^{n-1} \Delta_{2 k-1}(3)=A_{n}^{2}
$$

since

$$
\Delta_{2 k-1}(3)=\frac{(3+2 k)_{k-1}(2+2 k)_{k}}{(k)_{k}(2+k)_{k-1}}=\left(\frac{(3 k+1) ! k !}{(2 k) !(2 k+1) !}\right)^{2} .
$$

We also remark that other special values for $W_{n}(x)$ can be derived from Theorem 2 besides $\operatorname{det}\left(W_{n}(0)\right)=A_{n+1}^{2}$. Namely

$$
\begin{aligned}
& \operatorname{det}\left(W_{n}(-1)\right)=H_{2 n+1}, \\
& \operatorname{det}\left(W_{n}(-2)\right)=H_{2 n}, \\
& \operatorname{det}\left(W_{n}(-3)\right)=A_{n-1} A_{n},
\end{aligned}
$$

where the sequence $H_{n}$ is defined by $H_{0}=1$,

$$
\frac{H_{2 n+1}}{H_{2 n}}=\left(\begin{array}{c}
3 n \\
n
\end{array}\right) /\left(\begin{array}{c}
2 n \\
n
\end{array}\right), \quad \frac{H_{2 n}}{H_{2 n-1}}=\frac{4}{3} \frac{\left(\begin{array}{c}
3 n \\
n
\end{array}\right)}{\left(\begin{array}{c}
2 n \\
n
\end{array}\right)} \text {. }
$$

The sequences $A_{n}$ and $H_{n}$ occur in a number of unsolved problems of Mills-Robbins-Rumsey (cf. [11] for a survey of the problems).

6. Conclusion. The problem of enumerating symmetry classes of plane partitions is considered extensively in [12]. Indeed the identity (1.1) which we rely on heavily throughout our work was used by Mills et al. to treat plane partitions in a different symmetry class [9]. G. Kuperberg [7] has recently prepared an appealing survey of this topic. Also recently M. Ishikawa [6] has found a nice plane partition theoretic interpreation of $\operatorname{det}(v(n))=A_{n}^{2}$ (see (4.9) and (4.15)).

Acknowledgment. Every stage of this work and each theorem and lemma was found empirically using the symbolic algebra package AXIOM. While our proofs do not rely on the computer, each of our discoveries would have been impossible without the flexibility and power of AXIOM. 


\section{REFERENCES}

[1] G. E. Andrews, Plane partitions III: the weak Macdonald conjecture, Invent. Math., 53 (1979), 193-225.

[2] , Plane partitions V: the T.S.S.C.P.P. conjecture, J. Combin. Theory Ser. A, (to appear).

[3] W. N. Bailey, Some identities involving generalized hypergeometric series, Proc. London Math. Soc. Ser. 2, 29 (1929), 503-516.

[4] _ Generalized Hypergeometric Series (Reprinted: Hafner, New York, 1964), Cambridge University Press, London and New York, 1935.

[5] A. Erdelyi et al., Higher Transcendental Functions, vol. 1, McGraw-Hill, New York, 1953.

[6] M. Ishikawa, A remark on totally symmetric self-complementary plane partition, (to appear).

[7] G. Kuperberg, Symmetries of plane partitions and the permanent-determinant method, (to appear).

[8] W. H. Mills, D. P. Robbins and H. Rumsey, Self-complementary, totally symmetric plane partitions, J. Combin. Theory Ser. A, 42 (1986), 277-292.

[9] _ Enumeration of a symmetry class of plane partitions, Discrete Math., 67 (1987), 43-55.

[10] L. J. Slater, Generalized Hypergeometric Functions, Cambridge University Press, Cambridge, 1966.

[11] R. P. Stanley, A baker's dozen of conjectures concerning plane partitions, from Combinatoire énumérative (G. Labelle and P. Leroux, eds.), Lecture Notes in Math. No. 1234, Springer, Berlin/New York, 1986, pp. 285-293.

[12] __ Symmetries of plane partitions, J. Combin. Theory Ser. A, 3 (1986), 103113.

[13] J. Stembridge, Nonintersecting paths, Pfaffians, and plane partitions, Advances in Math., 83 (1990), 96-131.

[14] F. J. W. Whipple, Well-poised series and other generalized hypergeometric series, Proc. London Math. Soc. Ser. 2, 25 (1926), 525-544.

Received May 13, 1991 and in revised form March 16, 1992. Partially supported by NFS Grant DMS 8702695-03 and the IBM Thomas J. Watson Research Center.

Pennsylvania State University

UNIVERSITY PARK, PA 16802

AND

IBM Thomas J. Watson Research Center YORKTOWN HEIGHTS, NY 10598 


\title{
PACIFIC JOURNAL OF MATHEMATICS
}

Founded by

\author{
E. F. BeCKeNBACH (1906-1982) F. Wolf (1904-1989)
}

\section{EDITORS}

\section{S. VARADARAJAN (Managing Editor) University of California Los Angeles, CA 90024-1555 vsv@math.ucla.edu \\ F. Michael Christ University of California Los Angeles, CA 90024-1555 christ@math.ucla.edu}

\section{Herbert Clemens}

University of Utah

Salt Lake City, UT 84112

clemens@math.utah.edu

\author{
THOMAS ENRIGHT \\ University of California, San Diego \\ La Jolla, CA 92093 \\ tenright@ucsd.edu \\ Nicholas ERcolani \\ University of Arizona \\ Tucson, AZ 85721 \\ ercolani@math.arizona.edu \\ R. FINN \\ Stanford University \\ Stanford, CA 94305 \\ finn@gauss.stanford.edu \\ VAUGHAN F. R. JONES \\ University of California \\ Berkeley, CA 94720 \\ vfr@math.berkeley.edu
}

\section{SUPPORTING INSTITUTIONS}

UNIVERSITY OF ARIZONA

UNIVERSITY OF BRITISH COLUMBIA

UNIVERSITY OF OREGON

CALIFORNIA INSTITUTE OF TECHNOLOGY

UNIVERSITY OF CALIFORNIA

UNIVERSITY OF MONTANA

UNIVERSITY OF NEVADA, RENO

NEW MEXICO STATE UNIVERSITY

OREGON STATE UNIVERSITY
SteVen KerckhofF

Stanford University

Stanford, CA 94305

spk@gauss.stanford.edu

MARTIN SCHARLEMANN

University of California

Santa Barbara, CA 93106

mgscharl@henri.ucsb.edu

HAROLD STARK

University of California, San Diego

La Jolla, CA 92093

\author{
STANFORD UNIVERSITY \\ UNIVERSITY OF HAWAII \\ UNIVERSITY OF UTAH \\ WASHINGTON STATE UNIVERSITY \\ UNIVERSITY OF WASHINGTON
}

UIV 


\section{PACIFIC JOURNAL OF MATHEMATICS}

Volume $158 \quad$ No. $1 \quad$ March 1993

Determinant identities

GEORGE W. Eyre ANDrEWS and WiLliam H. Burge

A spectral theory for solvable Lie algebras of operators

E. BOASSO and ANGEL RAFAEL LAROTONDA

Simple group actions on hyperbolic Riemann surfaces of least area

S. Allen Broughton

Duality for finite bipartite graphs (with an application to $\mathrm{II}_{1}$ factors)

MARIE CHODA

Szegő maps and highest weight representations

MARK GREGORY DAVIDSON and Ron STANKE

Optimal approximation class for multivariate Bernstein operators

ZEEV DitZIAN and XINLONG ZHOU

Witt rings under odd degree extensions

ROBERT FITZGERALD

Congruence properties of functions related to the partition function

ANTHONY D. FORBES

Bilinear operators on $L^{\infty}(G)$ of locally compact groups

Colin C. GRAham and Anthony To-Ming LAU

Nonuniqueness of the metric in Lorentzian manifolds

GEOFFREY K. MARTIN and GERARD THOMPSON

Index theory and Toeplitz algebras on one-parameter subgroups of Lie 189 groups

EFTON PARK 